\title{
Vitamins C and E downregulate vascular VEGF and VEGFR-2 expression in apolipoprotein-E-deficient mice
}

\author{
Beatriz Nespereira $^{\mathrm{a}, 1}$, Maitane Pérez-Ilzarbe ${ }^{\mathrm{a}, 1}$, Patricia Fernández $^{\mathrm{b}}$, \\ Angela M. Fuentes ${ }^{c, 2}$, José A. Páramo ${ }^{a}$, José A. Rodríguez ${ }^{\mathrm{a}, *}$ \\ ${ }^{a}$ Atherosclerosis Research Unit, University of Navarra, C/Irunlarrea 1. CIFA, Pamplona E-31008, Spain \\ ${ }^{\mathrm{b}}$ Department of Ophthalmology, School of Medicine, University of Navarra, Pamplona, Spain \\ ${ }^{\mathrm{c}}$ Fundación Inbiomed, San Sebastián, Spain
}

Received 28 April 2003; received in revised form 11 August 2003; accepted 15 August 2003

\begin{abstract}
Anti-angiogenic therapy reduces both plaque growth and intimal neovascularization in apolipoprotein-E-deficient mice (apoE-/-). Vascular endothelial growth factor (VEGF) has been suggested as playing a role in the development of atherosclerosis. We examined the hypothesis that VEGF and VEGF receptor-2 (VEGFR-2) expression is upregulated in apoE-/- and, since it could be driven by oxidative stress, tested whether dietary supplementation with vitamins $\mathrm{C}$ and $\mathrm{E}$ could downregulate it.

Two-month-old apoE-/- received vitamin C combined with $\alpha$ - or $\beta$-tocopherol for 4 weeks. Aortic VEGF and VEGFR-2 expression were measured by RT-qPCR and western blot.

ApoE- $/-$ showed significantly higher expression of aortic VEGF and VEGFR-2 mRNA $(P<0.001)$ and protein $(P<0.001)$ than wild-type mice, as well as increased plasma VEGF $(P<0.001)$. Vitamin $\mathrm{C}$ and $\alpha$-tocopherol significantly reduced aortic VEGF and VEGFR-2 expression in apoE-I- $(P<0.001)$, circulating VEGF $(P<0.01)$ and plasma lipid peroxidation $(P<0.01)$. apoE-I- receiving vitamin $C$ and $\beta$-tocopherol showed diminished lipid peroxidation and VEGFR-2, but only partial reduction of VEGF expression.

These data demonstrate that augmented VEGF and VEGFR-2 expression in apoE-/- vasculature can be downregulated by vitamins C and E, at least partially through oxidative stress reduction. This novel mechanism could contribute to explaining the beneficial effects of antioxidant vitamins in experimental atherosclerosis.

(C) 2003 Elsevier Ireland Ltd. All rights reserved.
\end{abstract}

Keywords: VEGF; VEGFR-2; Hypercholesterolemia; Ascorbic acid; Tocopherol

\section{Introduction}

Vascular endothelial growth factor (VEGF), the main agent responsible for angiogenesis and vascular permeability, is a $45 \mathrm{kDa}$ glycoprotein secreted in the vascular wall by endothelial and smooth muscle cells. It has also been implicated in the maintenance of endothelial integrity, partly through upregulation of endothelial nitric oxide synthase

\footnotetext{
* Corresponding author. Tel.: +34-948-425600x6390; fax: +34-948-425652.

E-mail address: josean@unav.es (J.A. Rodríguez).

1 Beatriz Nespereira and Maitane Pérez-Ilzarbe equally contributed to this work.

2 Present address: Instituto de Estudios Avanzados, Baruta, Miranda, Venezuela.
}

expression [1]. VEGF expression is regulated mainly by hypoxia [2], oxidative stress [3] and nitric oxide [4,5]. The biological effects of VEGF are mediated mainly by two tyrosin kinase receptors: VEGFR-1 (Flt-1) and VEGFR-2 (flk-1/KDR). VEGFR-2 is essential for vasculogenesis, angiogenesis and hematopoiesis [6], and mediates VEGF antiapoptotic effects in endothelium [7].

Despite these functions, VEGF could also be considered a pro-atherogenic factor, because its administration increases the rate and degree of atherosclerotic plaque formation in apo-E/apo-B100 deficient mice and in hypercholesterolemic rabbits [8]. Moreover, VEGF has been found to be upregulated in human coronary atherosclerotic lesions but not in normal coronary segments [9]. Although the mechanism by which VEGF induces plaque formation has not been established, it has been suggested 
that new vessel formation causes plaque progression [8]. The administration of the anti-angiogenic drug endostatin to apolipoprotein-E-deficient mice (apoE-/-), a well-characterized model of atherosclerosis, reduces both plaque growth and intimal neovascularization, providing evidence that neovascularization is required for atherosclerosis plaque development [10].

Interestingly, the apoE-/- murine model of hypercholesterolemia is characterized by increased oxidative stress [11] and reduced nitric oxide bioavailability [12], two mechanisms known to contribute to atherosclerotic plaque development and also involved in the regulation of VEGF expression $[3,4]$. Increased VEGF levels have been reported in plasma from hypercholesterolemic individuals [13] and in coronary arteries from hypercholesterolemic pigs [14]. Moreover, it has been shown that oxidized LDL, a key molecule in the atherosclerotic process, can upregulate VEGF expression in macrophages and endothelial cells, at least partially through the activation of PPAR $\gamma$ [15]. In this context, assuming that VEGF acting through VEGFR-2 can be at least one of the factors responsible for the angiogenic processes required for atherosclerosis plaque development, we hypothesized that either of them could be upregulated in the vascular wall of apoE-/- mice.

A potential role of antioxidant vitamins in cardiovascular disease prevention has been proposed, based on the essential role of lipoproteins oxidation in the initiation and progression of atherosclerotic lesions. To date, there is no clear evidence from clinical trials that antioxidants provided by dietary intake or in the form of vitamin supplements can reduce the risk of atherosclerosis $[16,17]$. However, a number of studies in different animal models have considered the potential for different dietary antioxidants to help prevent development and progression of atherosclerosis, and most of the experimental evidence helps substantiate this hypothesis $[18,19]$. Particularly, $\alpha$-tocopherol has been shown to decrease lipid peroxidation and atherosclerosis in apoE-/[11].

Since VEGF and/or VEGFR-2 could be upregulated in apoE-/- vasculature as a consequence of increased oxidative stress, we investigated whether dietary supplementation with vitamin $C$ and $\alpha$-tocopherol could downregulate VEGF and VEGFR-2 expression in this animal model. The combination of vitamin $C$ and $\beta$-tocopherol was also studied to elucidate whether antioxidant mechanisms can fully account for the effects of vitamin supplementation.

\section{Methods}

\subsection{Mice}

C57BL/6 wild type (WT) mice and apoE-/- were procured from the colony at CIFA (GLP accredited center at the University of Navarra) animal facilities, originating from breeders obtained from Center for Transgene
Technology and Gene Therapy (Flanders Interuniversity Institute for Biotechnology, Leuven). The investigation was performed in accordance with the European Community guidelines for animal ethical care and use of laboratory animals (Directive 86/609), and was approved by the University of Navarra Animal Research Review Committee.

Two-month-old male wild type and apoE-/- mice fed a standard rodent diet (Harlan Teklad 9605) were divided into four groups $(n=11)$ : non-treated wild type and apoE-/-, and vitamins $\mathrm{C}$ and $\mathrm{E}$ supplemented mice (WT-CE, apoE-/-CE). Ascorbic acid $\left(120 \mathrm{mg} \mathrm{kg}^{-1}\right.$ per day) and $\alpha$-tocopherol $\left(210 \mathrm{mg} \mathrm{kg}^{-1}\right.$ per day) were given in drinking water. An additional group of apoE- $/-$ mice received $120 \mathrm{mg} \mathrm{kg}^{-1}$ per day ascorbic acid and $210 \mathrm{~m} \mathrm{~kg}^{-1}$ per day $\beta$-tocopherol $(n=5$, apoE-/-C $\beta E)$. Two hundred micromolar stock solutions of $\alpha$-tocopherol and $\beta$-tocopherol in ethanol were prepared to facilitate final dilutions in water. $\beta$-tocopherol was generously provided by Dr. Christine Gartner (Cognis, Germany). Daily liquid intake, measured by weighing water bottles, was similar in mice from each experimental group $(5 \mathrm{ml}$ per animal per day). After 4 weeks of treatment, animals were sacrificed and blood was drawn by cardiac puncture.

Plasma total cholesterol and tryglicerides were determined by colorometric assay (Sigma). Plasma concentration of thiobarbituric acid-reactive substances (TBARS) was measured, as indicator of lipid peroxidation [20]. Aorta was removed, dissected free of interstitial tissue with the help of a surgical microscope (Zeiss OPMI 6CR/S3), immediately frozen and stored at $-80^{\circ} \mathrm{C}$. Tocopherol concentration in plasma was measured by high performance liquid chromatography [19].

\subsection{RNA isolation and real-time quantitative RT-PCR}

Total RNA was extracted from each aorta with TRIzol (Invitrogene) and quantified with ultraviolet spectroscopy. Total RNA $(5 \mu \mathrm{g})$ was reverse-transcribed with oligo (dT) primers with M-MLV reverse transcriptase (Invitrogene), in the presence of SuperRNase In (Ambion).

Three hundred nanograms of reverse-transcribed RNA was primed with oligonucleotides specific for VEGF (5'-GAGACCCTGGTGGACATC-3' and 5'-TTTCTTTGGTCTGCATTC-3'), VEGFR-2 (5'-GACTGTGGCGAAGTGTTTTTGA-3' and 5'-GTGCAGGGGAGGGTTGGCGTAG- $3^{\prime}$ ) and $\beta$-actin ( $5^{\prime}$-GCCTTCCTTCTTGGGTATGG- $3^{\prime}$ and $5^{\prime}$-ACGCAGCTCAGTAACAGTCC- $3^{\prime}$ ). PCR was performed as previously described $\left(95^{\circ} \mathrm{C}\right.$ for $10 \mathrm{~min}$ and run for 40 cycles at $95^{\circ} \mathrm{C}$ for $15 \mathrm{~s}$ and $60^{\circ} \mathrm{C}$ for $1 \mathrm{~min}$ ) with SYBR Green PCR Master Mix (Applied Biosystems) on the ABI PRISM 5700 Detection System (Applied Biosystems) [21]. Potential genomic DNA contamination was excluded by using intron-encompassing primers. All samples were assayed in triplicate and normalized on the basis of their $\beta$-actin content. 


\subsection{Protein extraction, electrophoresis and western} blotting

Following TRIzol manufacturer's instructions, protein was extracted from aortic homogenates after RNA isolation. Protein concentration was determined according to Bradford [22]. VEGF was quantified in murine plasma and aorta by western blotting: plasma samples (diluted 1:20 in saline) and aortic homogenates ( $10 \mu \mathrm{g}$ total protein) were subjected to $12 \%$ SDS-PAGE, electroblotted onto nitrocellulose membranes and probed with anti-VEGF (1:1000, sc7269, Santa Cruz Biotechnologies), followed by a peroxidase-linked sheep anti-mouse IgG (1:5000, Amersham Biosciences). Blots were developed by enhanced chemiluminescence according to the manufacturer's instructions (ECL, Amersham Biosciences). Aortic expression of VEGFR-2 was assessed similarly, subjected to $6 \%$ SDS-PAGE, probed with anti-VEGFR-2 (1:1000, sc6251, Santa Cruz Biotechnologies) and developed by enhanced chemiluminescence (ECL Plus, Amersham Biosciences). Even loading was verified by Ponceau red staining.

\subsection{Statistical methods}

Data are presented as mean \pm standard deviation. Analysis of variance (ANOVA) or Kruskal-Wallis test was applied to assess differences between treatment groups. After a significant ANOVA or Kruskal-Wallis, comparisons were made with Bonferroni posthoc or Mann-Whitney test, respectively. A value of $P<0.05$ was accepted as statistically significant.

\section{Results}

\subsection{Cholesterol, triglyceride and lipid peroxidation in murine plasma}

Table 1 shows the general characteristics of the different animal groups. Plasma total cholesterol and triglycerides were significantly higher in apoE-/- than in WT mice
( $P<0.001$ in both cases). Both types of mice presented similar plasma tocopherol concentration, although tocopherol/cholesterol ratio was markedly reduced in apoE-/$(P<0.001)$, in agreement with the increased TBARS $(P<0.01)$, in comparison with WT mice.

\subsection{Vascular VEGF and VEGFR-2 expression in apoE-/- mice}

Vascular expression of VEGF mRNA in apoE-/- was nearly five times higher than in WT group $(P<0.001$; Fig. 1A). As shown in Fig. 1B, the observed mRNA increase was accompanied by a higher VEGF protein content in the vessel wall $(P<0.001)$.

Since VEGFR-2 plays a leading role in VEGF-induced angiogenesis, we analyzed vascular VEGFR-2 expression in the same in vivo model. As expected, a threefold increase in VEGFR-2 mRNA was observed in apoE-/- mice as compared with WT $(P<0.001$; Fig. 2A), and a higher VEGFR-2 protein content in the vessel wall could also be demonstrated ( $P<0.001$; Fig. 2B).

Relative VEGF levels in plasma were analyzed by western blotting to determine if they would reflect the observed changes in the vascular wall. VEGF plasma levels were significantly higher in apoE- - rather than WT mice $(P<$ 0.001; Fig. 3).

\subsection{Effect of vitamin $C$ and $\alpha$-tocopherol on VEGF and VEGFR-2}

Compliance with the vitamins supplementation was evident, as plasma tocopherol levels in the groups receiving vitamin $\mathrm{E}$ increased to above the levels in non-supplemented mice $(P<0.001)$, and significantly reduced apoE-/plasma TBARS $(P<0.01)$ down to WT levels, even though tocopherol/cholesterol ratio was still lower than WT $(P<0.01$; Table 1). While plasma total cholesterol and triglycerides remained unchanged after treatment in WT mice, a marginal reduction in total cholesterol levels was observed in treated apoE- $-(P<0.01)$, the levels still remaining eightfold higher than in WT.

Table 1

Plasmatic parameters of the different animal groups at the time of the sacrifice ${ }^{b}$

\begin{tabular}{|c|c|c|c|c|c|}
\hline & $\begin{array}{l}\text { Total cholesterol } \\
(\mathrm{mmol} / \mathrm{l})\end{array}$ & $\begin{array}{l}\text { Triglycerides } \\
(\mathrm{mmol} / \mathrm{l})\end{array}$ & $\begin{array}{l}\text { Tocopherol } \\
(\mu \mathrm{mol} / \mathrm{l})\end{array}$ & $\begin{array}{l}\text { Tocopherol/cholesterol } \\
(\mu \mathrm{mol} / \mathrm{mmol})\end{array}$ & $\begin{array}{l}\text { TBARS } \\
\text { (mmol MDA/l) }\end{array}$ \\
\hline $\mathrm{WT}(n=11)$ & $1.71 \pm 0.34$ & $0.60 \pm 0.26$ & $35.3 \pm 13.7$ & $18.1 \pm 7.1$ & $1.18 \pm 0.36$ \\
\hline WT-CE $(n=11)$ & $1.78 \pm 0.31$ & $0.53 \pm 0.12$ & $94.9 \pm 28.3^{* * *}$ & $50.2 \pm 13.2^{* * *}$ & $0.92 \pm 0.52$ \\
\hline apoE-l- $(n=11)$ & $18.78 \pm 4.04^{* * *}$ & $1.16 \pm 0.34^{* * *}$ & $36.8 \pm 12.5$ & $1.9 \pm 0.7^{* * *}$ & $1.76 \pm 0.45^{* * *}$ \\
\hline apoE-/-CE $(n=11)$ & $14.74 \pm 3.78^{* * * \dagger \dagger}$ & $1.04 \pm 0.15^{* * *}$ & $103.4 \pm 34.5^{\dagger \dagger \dagger}$ & $7.4 \pm 2.7^{* * \dagger \dagger}$ & $1.25 \pm 0.39^{\dagger \dagger}$ \\
\hline apoE-/-C $\beta E(n=5)$ & $18.83 \pm 2.38^{* * *}$ & $0.88 \pm 0.10^{* * *}$ & $100.7 \pm 46.7^{\dagger \dagger}$ & $7.5 \pm 3.5^{* * \dagger \dagger}$ & $1.14 \pm 0.16^{\dagger \dagger}$ \\
\hline
\end{tabular}

Plasma total cholesterol (mmol/l), triglycerides (mmol/l), tocopherol ( $\mu$ mol/l) and TBARS (mmol MDA/l) are shown. Statistically significant differences from WT are marked as ${ }^{* *}(P<0.01),{ }^{* * *}(P<0.001)$, and from apoE-l- as $\dagger^{\dagger \dagger}\left(P<0.01,{ }^{\dagger \dagger}(P<0.001)\right.$.

b WT: wild type; WT-CE: supplemented with vitamins C and E; apoE-/-: apoE-deficient; apoE-/-CE: apoE-/- supplemented with vitamin C and apoE-/-C $\beta E$ : $\alpha$-tocopherol or $\beta$-tocopherol. 

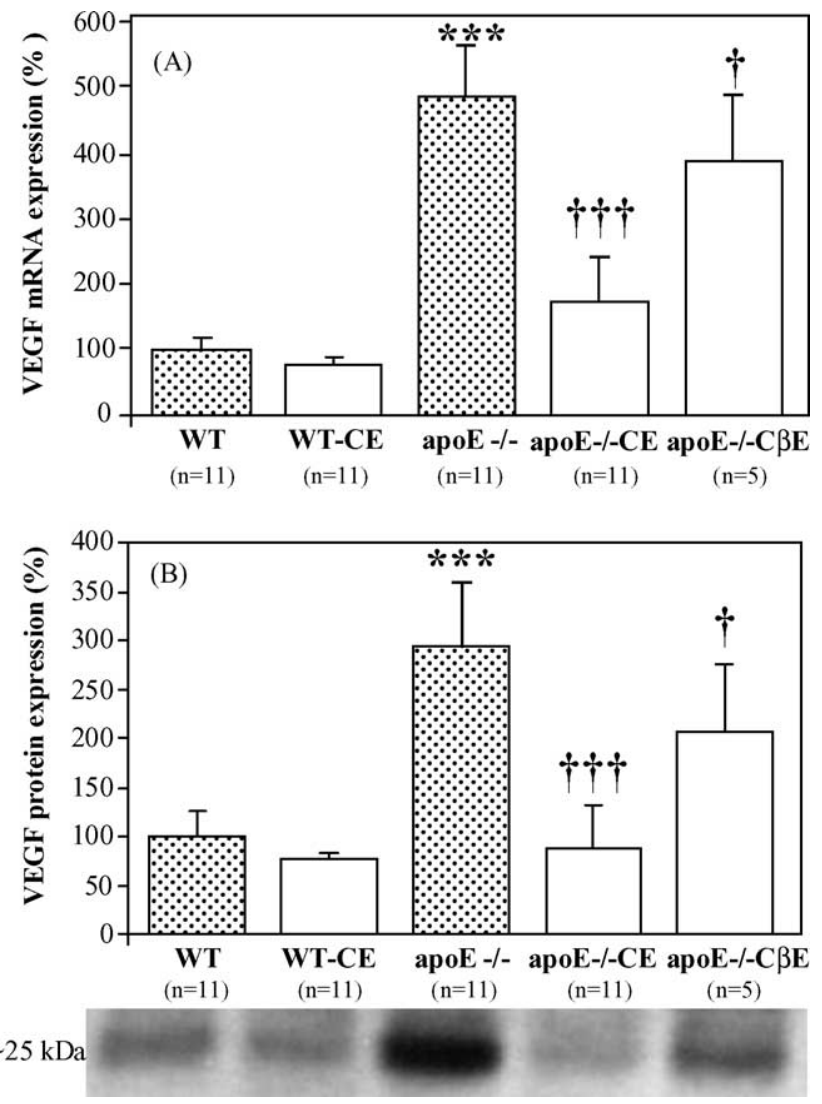

Fig. 1. Changes in vascular VEGF expression (mRNA and protein), reported as percentage increase with respect to untreated wild type (WT) mice. (A) VEGF mRNA, assessed by RT-qPCR and corrected by $\beta$-actin mRNA content, is increased in apoE-/- mice. Vitamin $\mathrm{C}$ $+\alpha$-tocopherol treatment (apoE-/-CE) reduces it significantly, but vitamin $C+\beta$-tocopherol (apoE-/ $-\mathrm{C} \beta E$ ) only achieves a partial reduction. (B) Augmented VEGF protein in apoE-/-, assessed by western blot (densitometric analysis and representative blot showing the $25 \mathrm{kDa}$ VEGF monomer), is significantly reduced in apoE $-/-\mathrm{CE}$ and apoE- $-/ \mathrm{C} \beta \mathrm{E}$. Bars represent the mean \pm S.D. for all the animals in the experimental group (size indicated below $x$-axis). Statistically significant differences from WT are marked as ${ }^{* * *}(P<0.001)$, and from apoE $-I-$ as $\dagger(P<0.05),{ }^{\dagger \dagger \dagger}(P<0.001)$.

Interestingly, the combination of vitamin $\mathrm{C}$ and $\alpha$ tocopherol significantly decreased aortic VEGF and VEGFR-2 expression in apoE-/-, both at mRNA and protein level $(P<0.001$ in all cases; Figs. 1 and 2$)$, but it did not substantially modify their expression in WT. It only showed a marginal reduction in VEGF and VEGFR-2 mRNA expression remaining unchanged.

As found in the vessel wall, vitamin $\mathrm{C}$ and $\alpha$-tocopherol also markedly reduced VEGF plasma levels in apoE-/mice (Fig. $3, P<0.01$ ), as compared with WT.

\subsection{Effect of vitamins $C$ and $\beta$-tocopherol on VEGF and VEGFR-2}

Dietary supplementation of apoE-/- mice with ascorbic acid and $\beta$-tocopherol was equally efficient to increase plasma tocopherol concentration $(P<0.01)$ and to reduce
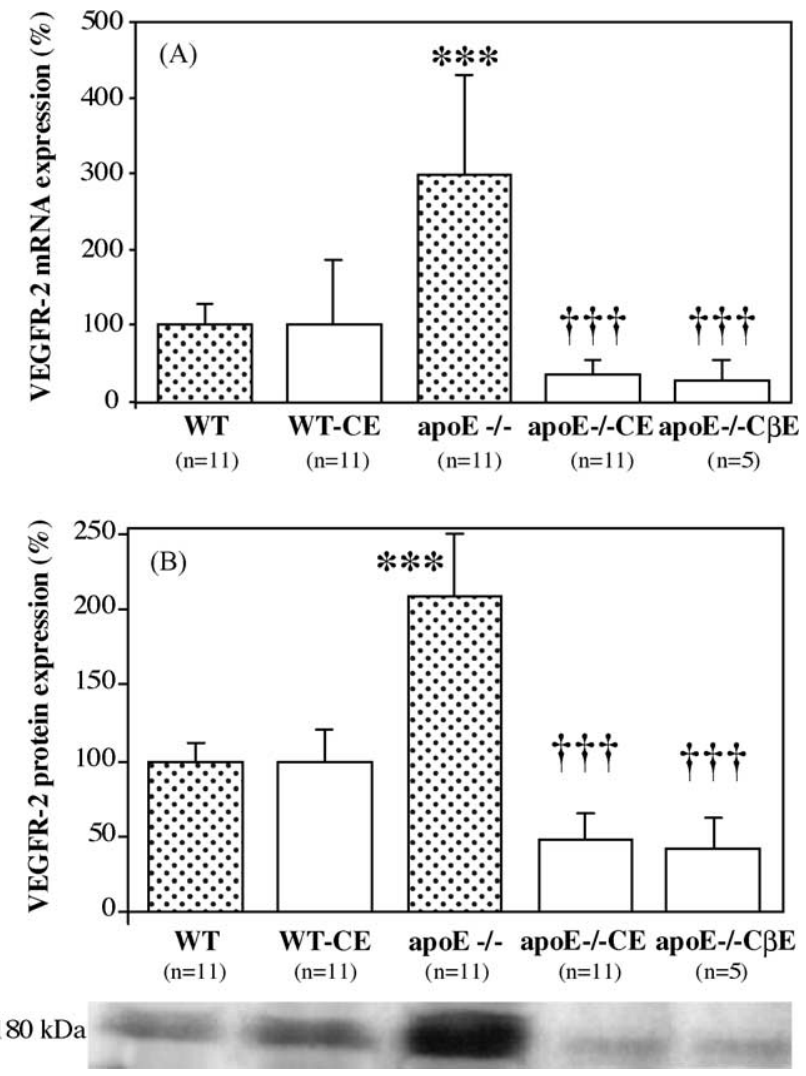

Fig. 2. Vascular VEGFR-2 expression, reported as percentage increase with respect to untreated wild type (WT) mice. (A) VEGFR-2 mRNA, assessed by RT-PCR and corrected by $\beta$-actin mRNA content, is increased in apoE-/- mice. Vitamin $\mathrm{C}$ combined with $\alpha$-tocopherol (apoE-/-CE) or $\beta$-tocopherol (apoE- $-\mathrm{C} \beta \mathrm{E}$ ) reduces it significantly. (B) Augmented VEGFR-2 protein in apoE-/-, assessed by western blot (densitometric analysis and representative blot), is significantly reduced in apoE-/-CE and apoE-/ $-\mathrm{C} \beta E$. Bars represent the mean \pm S.D. for all the animals in the experimental group (size indicated below $x$-axis). Statistically significant differences from WT are marked as ${ }^{* * *}(P<0.001)$, and from apoE- $/-$ as $\dagger^{\dagger \dagger}(P<0.001)$.

plasma TBARS $(P<0.01)$, the tocopherol/cholesterol ratio being still lower than WT $(P<0.01$; Table 1$)$. No modifications were observed in total cholesterol or triglyceride concentrations.

In contrast to mice receiving vitamin $\mathrm{C}$ and $\alpha$-tocopherol, treatment of apoE- $/$ - with vitamin $\mathrm{C}$ and $\beta$-tocopherol only achieved a partial, although significant, reduction (20\%) in vascular VEGF mRNA and protein expression $(P<0.05$, Fig. 1). However, no differences between the effect of $\alpha$ and $\beta$-tocopherol were observed in plasma VEGF (Fig. 3) or in vascular VEGFR-2 mRNA and protein levels (Fig. 2).

No deleterious effects were observed in any of the animals receiving the different vitamin treatments.

\section{Discussion}

We report herein that vitamins $\mathrm{C}$ and $\mathrm{E}$ markedly reduce the expression of VEGF and its endothelial receptor 


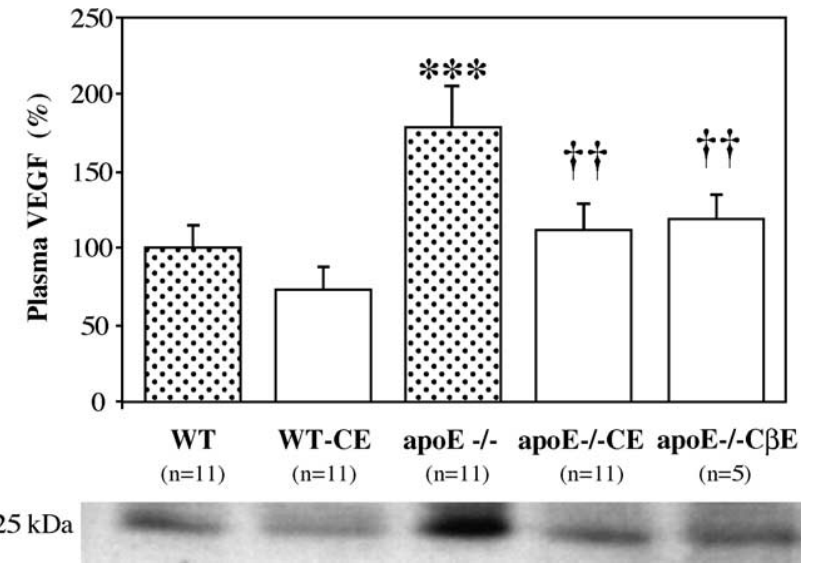

Fig. 3. Relative VEGF concentration in plasma, assessed by western blotting, and reported as percentage increase with respect to untreated wild type (WT) animals. Increased circulating VEGF in apoE-/- mice can be reduced by vitamin $\mathrm{C}+\alpha$-tocopherol (apoE-/-CE) or $\beta$-tocopherol (apoE-/-C $\beta E$ ) treatment. Lower panel: representative immunoblot of murine plasma. Bars represent the mean \pm S.D. for all the animals in the experimental group (size indicated below $x$-axis). Statistically significant differences from WT are marked as ${ }^{* * *} P<0.001$, and from apoE-/as $\dagger^{\dagger} P<0.01$.

VEGFR-2 in a murine model of atherosclerosis, representing a novel effect of these compounds. In this study, we demonstrate a significant increase of VEGF and VEGFR-2 in the aorta of apoE- - mice, as compared to WT. Furthermore, to our knowledge, this is the first report showing that dietary supplementation with vitamins $\mathrm{C}$ and $\mathrm{E}$ significantly reduces vascular expression of VEGF and VEGFR-2 in this experimental model.

VEGF has a dual role in vascular homeostasis: it is not only the principal agent responsible for angiogenesis, vascular permeability and maintenance of endothelial integrity, but it also favors the progression and growth of the atherosclerotic plaque [8]. VEGF and VEGFR-2 expression were measured in apoE-/- mice, an accepted model of atherosclerosis showing high cholesterol concentration, increased lipid peroxidation [11] and low nitric oxide bioavailability [12]. We found higher levels of aorta and plasma VEGF in apoE-/- than in WT, in agreement with previous observations in the coronary arteries of hypercholesterolemic pigs [14], as well as in the plasma of hypercholesterolemic patients [13]. Moreover, increased vascular VEGF was accompanied by a marked upregulation in VEGFR-2 expression, a novel finding not described previously in hypercholesterolemia.

Upregulation of VEGF and VEGFR-2 in the vessel wall could account for the increased vascular permeability [23] and enhanced vasa vasorum neovascularization observed in experimental models of hypercholesterolemia [24]. Although the mechanism by which hypercholesterolemia may induce VEGF upregulation in the vessel wall remains unclear, possible pathophysiological stimuli include hypoxia [14], oxidative stress [3] and low NO bioavailability [4]. Moreover, VEGF upregulation has been proposed to be a non-specific stress-induced vascular response in the adult organism [25], or to constitute a vascular homeostatic mechanism for compensating endothelial dysfunction [1]. Independently of the mechanism responsible, sustained vascular VEGF overexpression could contribute to the atherosclerotic process by favoring lipoprotein infiltration through increased vascular permeability [23], inflammation [26] and angiogenesis [8]. The anti-atherosclerotic effect of the angiogenesis inhibitors endostatin and TNP-470 in the apoE-/- [10] would support this hypothesis.

Dietary supplementation of apoE-/- mice with ascorbic acid and $\alpha$-tocopherol significantly decreased both VEGF and VEGFR-2 expression, as well as plasma TBARS, while it augmented the tocopherol/cholesterol ratio. It can be speculated that the observed downregulation could be due to their antioxidant activity and/or the protein kinase $\mathrm{C}$ (PKC) inhibitory activity of $\alpha$-tocopherol. A similar vitamin $\mathrm{E}$ dose, higher than that used therapeutically in humans, has been proved to reduce oxidative stress and atherosclerosis in apoE-/- mice [11], and vitamin $\mathrm{C}$ was administered to ensure $\alpha$-tocopherol regeneration from $\alpha$-tocopheroxy radical, thereby preventing vitamin $\mathrm{E}$ pro-oxidant activity and tocopherol-mediated peroxidation [27]. In spite of the high dose of vitamin E administered, the threefold increase observed in plasma tocopherol is not far away from the twofold increase reported in several human trials studying vitamin E supplementation.

The combination of ascorbic acid and $\beta$-tocopherol, an equally strong antioxidant in comparison to $\alpha$-tocopherol but lacking other biological properties present in $\alpha$-tocopherol PKC inhibitory and antiproliferative activities) [28] partially decreased VEGF expression, suggesting that the reduction of oxidative stress is not the only mechanism involved. Since augmented PKC activity has been shown in apoE-/- [29] and PKC activation can upregulate VEGF expression [4], it is conceivable that the observed in vivo effect of $\alpha$-tocopherol on VEGF expression is due to both antioxidant and PKC inhibitory effects. It could be also argued that differences in the intestinal absorption, translated into different bioavailabilities for $\alpha$ and $\beta$-tocopherol, could account for the observed effects on VEGF expression. However, no differences were observed in plasma tocopherol between vitamin-treated apoE- $-1-$ mice, and the fact that both treatments similarly reduced vascular VEGFR-2 expression suggests that antioxidant mechanisms are fully responsible for VEGFR-2 downregulation while only accounting for partial VEGF reduction. This point would require further confirmation by measuring local production of reactive oxygen species and/or lipid peroxidation markers more specific and sensitive than TBARS (i.e. isoprostanes).

Interestingly, both treatments also achieved a similar significant reduction in circulating VEGF levels. Although the pathophysiological meaning of the observed changes is not clear yet [13], given that systemic VEGF levels may not strictly reflect changes occurring in the vessel wall, its determination could become a valuable tool to investigate the 
relationship between hypercholesterolemia, atherosclerosis and angiogenesis, together with its pharmacological modulation.

Since VEGF seems to be necessary in the development of atherosclerosis [26] and treatment with angiogenesis inhibitors reduces both plaque growth and intimal neovascularization in apoE-/- mice [10], the observed downregulation of VEGF and VEGFR-2 expression by the combination of vitamins $\mathrm{C}$ and $\mathrm{E}$ would be an additional mechanism by which these agents could prevent and limit atherosclerotic plaque progression $[18,30]$. Further studies should be conducted in a well-defined human hypercholesterolemic population to assess whether the effects of vitamins $\mathrm{C}$ and E supplementation observed in mice can be reproduced also in humans.

\section{Acknowledgements}

We thank Diego Martínez-Caro for his continuous support and Inés Montero for HPLC measurements. This study was partially supported by grants from the Department of Education and Culture (Government of Navarra, Pamplona, Spain) and Spanish Society of Cardiology (Madrid, Spain) to JAR. BN, MPI and PF have received educational grants from the Department of Education and Culture (Government of Navarra, Pamplona, Spain).

\section{References}

[1] Zachary I, Mathur A, Yla Herttuala S, Martin J. Vascular protection: A novel nonangiogenic cardiovascular role for vascular endothelial growth factor. Arterioscler Thromb Vasc Biol 2000;20(6):1512-20.

[2] Brogi E, Wu T, Namiki A, Isner JM. Indirect angiogenic cytokines upregulate VEGF and bFGF gene expression in vascular smooth muscle cells, whereas hypoxia upregulates VEGF expression only. Circulation 1994;90(2):649-52.

[3] Chua CC, Hamdy RC, Chua BH. Upregulation of vascular endothelial growth factor by $\mathrm{H}_{2} \mathrm{O}_{2}$ in rat heart endothelial cells. Free Radic Biol Med 1998;25(8):891-7.

[4] Tsurumi Y, Murohara T, Krasinski K, Chen D, Witzenbichler B, Kearney M, et al. Reciprocal relation between VEGF and NO in the regulation of endothelial integrity. Nat Med 1997;3(8):879-86.

[5] Jozkowicz A, Cooke JP, Guevara I, Huk I, Funovics P, Pachinger $\mathrm{O}$, et al. Genetic augmentation of nitric oxide synthase increases the vascular generation of VEGF. Cardiovasc Res 2001;51(4):773-83.

[6] Shalaby F, Ho J, Stanford WL, Fischer KD, Schuh AC, Schwartz L, et al. A requirement for Flk1 in primitive and definitive hematopoiesis and vasculogenesis. Cell 1997;89(6):981-90.

[7] Gerber HP, McMurtrey A, Kowalski J, Yan M, Keyt BA, Dixit $\mathrm{V}$, et al. Vascular endothelial growth factor regulates endothelial cell survival through the phosphatidylinositol $3^{\prime}$-kinase/Akt signal transduction pathway. Requirement for Flk-1/KDR activation. J Biol Chem 1998;273(46):30336-43.

[8] Celletti FL, Waugh JM, Amabile PG, Brendolan A, Hilfiker PR, Dake MD. Vascular endothelial growth factor enhances atherosclerotic plaque progression. Nat Med 2001;7(4):425-9.

[9] Inoue $\mathrm{M}$, Itoh $\mathrm{H}$, Ueda $\mathrm{M}$, Naruko $\mathrm{T}$, Kojima A, Komatsu R, et al. Vascular endothelial growth factor (VEGF) expression in human coronary atherosclerotic lesions: possible pathophysiological significance of VEGF in progression of atherosclerosis. Circulation 1998;98(20):2108-16.

[10] Moulton KS, Heller E, Konerding MA, Flynn E, Palinski W, Folkman J. Angiogenesis inhibitors endostatin or TNP-470 reduce intimal neovascularization and plaque growth in apolipoprotein-E-deficient mice. Circulation 1999;99(13):1726-32.

[11] Pratico D, Tangirala RK, Rader DJ, Rokach J, FitzGerald GA. vitamin E suppresses isoprostane generation in vivo and reduces atherosclerosis in ApoE-deficient mice. Nat Med 1998;4(10):1189-92.

[12] Godecke A, Ziegler M, Ding Z, Schrader J. Endothelial dysfunction of coronary resistance vessels in apoE-/- mice involves NO but not prostacyclin-dependent mechanisms. Cardiovasc Res 2002;53(1):253-62.

[13] Blann AD, Belgore FM, Constans J, Conri C, Lip GY. Plasma vascular endothelial growth factor and its receptor Flt-1 in patients with hyperlipidemia and atherosclerosis and the effects of fluvastatin or fenofibrate. Am J Cardiol 2001;87(10):1160-3.

[14] Wilson S, Herrmann J, Lerman LO, Holmes DR, Napoli C, Ritman EL, et al. Simvastatin preserves the structure of coronary adventitial vasa vasorum in experimental hypercholesterolemia independent of lipid lowering. Circulation 2002;105(4):415-8.

[15] Inoue M, Itoh H, Tanaka T, Chun TH, Doi K, Fukunaga Y, et al. Oxidized LDL regulates vascular endothelial growth factor expression in human macrophages and endothelial cells through activation of peroxisome proliferator-activated receptor-gamma. Arterioscler Thromb Vasc Biol 2001;21(4):560-6.

[16] Lonn E. Do antioxidant vitamins protect against atherosclerosis? The proof is still lacking. J Am Coll Cardiol 2001;38(7):1795-8.

[17] Brigelius-Flohe R, Kelly FJ, Salonen JT, Neuzil J, Zingg JM, Azzi A. The European perspective on vitamin E: current knowledge and future research. Am J Clin Nutr 2002;76(4):703-16.

[18] Carr AC, Zhu BZ, Frei B. Potential antiatherogenic mechanisms of ascorbate (vitamin C) and alpha-tocopherol (vitamin E). Circ Res 2000;87(5):349-54.

[19] Rodriguez JA, Grau A, Eguinoa E, Nespereira B, Perez-Ilzarbe M, Arias R, et al. Dietary supplementation with vitamins $\mathrm{C}$ and $\mathrm{E}$ prevents downregulation of endothelial NOS expression in hypercholesterolemia in vivo and in vitro. Atherosclerosis 2002;165(1):33-40.

[20] Conti M, Morand P, Levillain P, Lemonnier A. Improved fluorometric determination of malonaldehyde. Clin Chem 1991;37(7):1273-5.

[21] Mendoza L, Carrascal T, De Luca M, Fuentes AM, Salado C, Blanco $\mathrm{J}$, et al. Hydrogen peroxide mediates vascular cell adhesion molecule-1 expression from interleukin-18-activated hepatic sinusoidal endothelium: implications for circulating cancer cell arrest in the murine liver. Hepatology 2001;34(2):298-310.

[22] Bradford MM. A rapid and sensitive method for the quantitation of microgram quantities of protein utilizing the principle of protein-dye binding. Anal Biochem 1976;72:248-54.

[23] Rodriguez Porcel M, Lerman A, Best PJ, Krier JD, Napoli C, Lerman LO. Hypercholesterolemia impairs myocardial perfusion and permeability: role of oxidative stress and endogenous scavenging activity. J Am Coll Cardiol 2001;37(2):608-15.

[24] Kwon HM, Sangiorgi G, Ritman EL, McKenna C, Holmes DR, Schwartz RS, et al. Enhanced coronary vasa vasorum neovascularization in experimental hypercholesterolemia. J Clin Invest 1998;101(8):1551-6.

[25] Clauss M, Schaper W. Vascular endothelial growth factor: a jack-ofall-trades or a nonspecific stress gene? Circ Res 2000;86(3):251-2.

[26] Zhao Q, Egashira K, Inoue S, Usui M, Kitamoto S, Ni W, et al. Vascular endothelial growth factor is necessary in the development of arteriosclerosis by recruiting/activating monocytes in a rat model of long-term inhibition of nitric oxide synthesis. Circulation 2002;105(9):1110-5.

[27] Neuzil J, Thomas SR, Stocker R. Requirement for, promotion, or inhibition by alpha-tocopherol of radical-induced initiation of plasma lipoprotein lipid peroxidation. Free Radic Biol Med 1997;22(1/2):57_ 71. 
[28] Tasinato A, Boscoboinik D, Bartoli GM, Maroni P, Azzi A $\mathrm{D}-\alpha$-tocopherol inhibition of vascular smooth muscle cell proliferation occurs at physiological concentrations, correlates with protein kinase $\mathrm{C}$ inhibition and is independent of its antioxidant properties. Proc Natl Acad Sci USA 1995;92(26):12190-4.

[29] Rosenblat M, Aviram M. Oxysterol-induced activation of macrophage NADPH-oxidase enhances cell-mediated oxidation of LDL in the atherosclerotic apolipoprotein-E-deficient mouse: inhibitory role for vitamin E. Atherosclerosis 2002;160(1):69-80.

[30] Cyrus T, Yao Y, Rokach J, Tang LX, Pratico D. vitamin E reduces progression of atherosclerosis in low-density lipoprotein receptor-deficient mice with established vascular lesions. Circulation 2003;107(4):521-3 